\title{
Dynamics of the thermocline in the equatorial region of the Pacific ocean
}

Calin Iulian Martin

To cite this article: Calin Iulian Martin (2015) Dynamics of the thermocline in the equatorial region of the Pacific ocean, Journal of Nonlinear Mathematical Physics 22:4, 516-522, DOI: https://doi.org/10.1080/14029251.2015.1113049

To link to this article: https://doi.org/10.1080/14029251.2015.1113049

Published online: 04 January 2021 


\title{
Dynamics of the thermocline in the equatorial region of the Pacific ocean
}

\author{
Calin Iulian Martin \\ Fakultät für Mathematik, Universität Wien, \\ Oskar-Morgenstern-Platz 1, 1090 Wien, Austria. \\ calin.martin@univie.ac.at
}

Received 25 August 2015

Accepted 5 October 2015

\begin{abstract}
This paper is devoted to the subsurface current dynamics in equatorial regions, where the hallmark of a strong stratification is a sharp interface (thermocline), separating two layers of different density, and whose depth is dependent upon the strength of the winds above the ocean's surface. We give here a few monotonicity results concerning the dynamics of the thermocline in the equatorial region. The most important one asserts that the level of the thermocline decreases as the strength of the wind at ten meters above the ocean surface, denoted $\left|U_{w}\right|$, increases. Moreover, the strength of the current at the thermocline decreases as $\left|U_{w}\right|$ increases.
\end{abstract}

Keywords: Ocean flows; Equatorial Undercurrent

2000 Mathematics Subject Classification: 35Q31, 35Q35

\section{Introduction}

We study the dynamics of waves in the zone of the Pacific Ocean situated within a band about $2^{\circ}$ latitude from the Equator. This area presents several peculiarities due to the pronounced stratification, cf. [11], the presence of underlying non-uniform currents and the existence of a diversity of ocean flows. The first aspect, concerning the stratification is stressed by the existence of a sharp nearsurface pycnocline/thermocline which separates a shallow layer of warm water, near the surface, from a deeper layer of colder, denser water-the difference in density being about $1 \%$. Concerning the existence of underlying non-uniform currents, it is known, cf. [20] and [21], that in a strip of approximately $300 \mathrm{~km}$ wide about the Equator, the underlying currents exhibit a pronounced depthdependence. Namely, in a layer adjacent to the surface, of about $100 \mathrm{~m}$, there is a westward drift that is triggered by the prevailing trade winds. Right below this layer lies the Equatorial Undercurrent (EUC), an eastward pointing stream which is essentially based on the thermocline. As far as our paper is concerned, we aim to investigate here the interactions between the two opposed currents as described earlier. To this end, we consider an initially calm setting that undergoes a sudden westward wind-stress acting on the ocean surface and which, afterwards remains constant for a long time. Moreover, assuming a constant ocean depth, no land boundaries and a uniform wind stress, it is matter of fact that, cf. [10] no see surface elevation will arise. However, the initial constant pressure at the ocean surface is modified and an underlying current field occurs. It is observed, for instance, in the context of the El Niño phenomenon that the pressure at the surface undergoes a rapid adjustment to the constant atmospheric pressure after the wind softens. The situation just described concerning the free surface and the pressure sets up the scenario that will be relevant to our endeavor. 


\section{Equations of motion and boundary conditions}

\subsection{Equations of motions}

Suitable for our context, described in the introduction, is the $f$-plane approximation for the governing equations, cf. $[6,19]$ and the considerations in [2]. We point out that the latter mentioned approximation is currently used extensively in investigations of equatorial fluid flows, cf. the discussion in the recent papers $[7,16,17]$. We also mention that the $f$-plane approximation is applicable in regions close to the Equator, where the ocean dynamics acquires peculiar features, usually captured within the broader framework of the $\beta$-plane approximation, discussed in detail in the survey papers [12,14], in the book [9] and in the recent papers [3-5, 15].

We will use a coordinate system with the origin at a point on the Earth's surface, with the $x$-axis chosen horizontally due East, the $y$-axis horizontally due North (in the tangent plane) and the $z$-axis oriented upward.

Assuming a simplified structure of the westward trade winds, a uniform wind stress, a vanishing fluid velocity and considering the linearized equations for a forced steady-state flow, the governing equations in the $f$-plane approximation in the layer adjacent to the free surface above the thermocline $z=-h$ are

$$
\begin{gathered}
0=-\frac{1}{\rho} P_{x}+\left(v u_{z}\right)_{z}, \\
-2 \Omega u=-\frac{1}{\rho} P_{z}-g, \\
u_{x}=0,
\end{gathered}
$$

where $P$ denotes pressure, $\rho$ represents the density, $\Omega$ is the rotational speed of the Earth and $v(z)$ is the vertical viscosity parameter, assumed here to be depth dependent, feature that is in contrast with the classical model of uniform vertical viscosity, see [22].

In the layer below the thermocline the motion of the flow is governed by

$$
\begin{gathered}
0=-\frac{1}{(1+r) \rho} P_{x}+\left(v u_{z}\right)_{z}, \\
-2 \Omega u=-\frac{1}{(1+r) \rho} P_{z}-g, \\
u_{x}=0,
\end{gathered}
$$

where $r$ is a small positive constant accounting for the difference in density between the two layers. Its values are typically between $4 \times 10^{-3}$ and $6 \times 10^{-3}$, cf. [11] and [18]. Moreover, according to McCreary [20] the stress-shear relationship

$$
\tau=\rho v u_{z}
$$

for the wind-induced stress $\tau(z)$ holds within the fluid. 


\subsection{Boundary and interface conditions}

One of the boundary conditions refers to the knowledge of the surface wind stress, $\tau_{0}<0$, thus, as a consequence of (2.3) we have

$$
\left.\rho v u_{z}\right|_{z=0}=\tau_{0}
$$

Following the arguments in [23] we assume that $v(0)=\sigma\left|\tau_{0}\right|$, for some (dimensional) constant $\sigma$. From the latter equation we have

$$
\left.u_{z}\right|_{z=0}=-\frac{1}{\rho \sigma} .
$$

The boundary conditions associated to the layer below the thermocline refer to the continuity of the horizontal velocity component, of the pressure and of the shear stress across the thermocline, and the no-slip condition

$$
u=0 \quad \text { on the ocean bed } \quad z=-d .
$$

Differentiating equation (2.1a) with respect to $z$, equation (2.1b) with respect to $x$ and taking into account $(2.1 \mathrm{c})$ we find that

$$
\left(v u_{z}\right)_{z z}=0 \text { for }-h \leq z \leq 0 .
$$

Thus, using the assumption of vanishing shear at the thermocline and relation (2.4), we find that

$$
v u_{z}=-\frac{v(0)}{\rho \sigma}\left(1+\frac{z}{h}\right) \quad \text { for } \quad-h \leq z \leq 0 .
$$

We infer from the previous relation that

$$
u(z)=u(0)+\frac{v(0)}{\rho \sigma} \int_{z}^{0} \frac{1+\frac{s}{h}}{v(s)} d s \text { for } \quad-h \leq z \leq 0 .
$$

Remark 2.1. Between the wind speed at 10 meters above the sea, denoted $U_{w}$, and the speed of the flow at the free surface $u(0)=: u_{0}$, there exists, cf. [1,13], the following relation

$$
U_{w}=\frac{1}{k} u_{0} \ln \left(\frac{10 g}{a u_{0}^{2}}+1\right),
$$

where $k \cong 0.4$ is the Kárman constant and $a \cong 0.0185$ is also a constant. Since the wind blows from east to west $U_{w}<0$, thus $u_{0}$ is also negative, as it follows from (2.8).

From the dynamic boundary condition at the free surface we obtain

$$
P(x, z)=-\frac{v(0)}{\sigma h} x-\rho g z-2 \rho \Omega \int_{z}^{0} u(s) d s+P_{a t m} \quad \text { for } \quad-h \leq z \leq 0 .
$$

Using now the equations in (2.2) we can infer that

$$
\left(v u_{z}\right)_{z z}=0
$$

in the layer below the thermocline. The assumption of vanishing shear along the thermocline yields

$$
v u_{z}=C\left(1+\frac{z}{h}\right) \quad \text { for } \quad-d \leq z \leq-h
$$


for some constant $C$ that is to be determined from the interface conditions along the thermocline in the following way. Since $P$ is continuous along the thermocline $z=-h$ we have that also $P_{x}$ is continuous across it. Thus, using $\left(v u_{z}\right)_{z}=\frac{C}{h}$, formula (2.9) and the first equation in (2.2) we find that

$$
C=-\frac{v(0)}{(1+r) \rho \sigma}
$$

and consequently

$$
u(z)=-\frac{v(0)}{(1+r) \rho \sigma} \int_{-d}^{z} \frac{1+\frac{s}{h}}{v(s)} d s \text { for } \quad-d \leq z \leq-h,
$$

where we have also made use of formula (2.5). Moreover, from (2.2) we obtain that for the pressure in the layer below the thermocline we have the formula

$$
P(x, z)=-\frac{v(0)}{\sigma h} x-\rho g[(1+r) z+r h]-2 \rho \Omega \int_{-h}^{0} u(s) d s-2 \rho \Omega(1+r) \int_{z}^{-h} u(s) d s+P_{a t m}
$$

From the continuity of the horizontal velocity component along the thermocline we have

$$
u(0)+\frac{v(0)}{\rho \sigma} \int_{-h}^{0} \frac{1+\frac{s}{h}}{v(s)} d s=-\frac{v(0)}{(1+r) \rho \sigma} \int_{-d}^{-h} \frac{1+\frac{s}{h}}{v(s)} d s .
$$

From the already derived formulas a few remarks are in order. Concerning the monotonicity of the horizontal velocity $u$ we have the following

Remark 2.2. From (2.6) we see that $u$ is a decreasing function in the layer above the thermocline, thus, as the depth decreases from $z=0$ to $z=-h$ the value of $u$ increases from the negative $u_{0}$ to the positive value $u(-h)$. From (2.10) we notice that $u$ is increasing in the layer below the thermocline, thus, as the depth increases from $z=-d$ to $z=-h$, the value of $u$ increases from $u(-d)=0$ to $u(-h)>0$. Consequently, $u$ achieves its minimum at the free surface and its maximum at the interface. See figure Figure 1 for a better understanding of the monotonicity of the equatorial undercurrent.

Remark 2.3. Formula (2.12) in conjunction with (2.8) indicates an implicit dependence of the thermocline depth, $-h$, on the wind speed $U_{w}$. We will pursue this dependence in greater detail for special choices of the viscosity function $v$.

It is known, cf. Cronin and Kessler, [8], that for moderate and high wind speeds a good choice of the viscosity function is

$$
v(z)=v(0) f\left(\frac{z}{d}\right), \quad \text { for } \quad-d \leq z \leq 0,
$$

for a (non-dimensional) function $f$ that decays exponentially with depth in the layer above the thermocline. We will prove in the sequel a monotonicity result concerning the level of the thermocline with respect to the strength of $U_{w}$.

Proposition 2.1. We assume that the viscosity coefficient $v$ is of the form described in (2.13). Then the level of the thermocline, $-h$, decreases as the strength of the wind speed (typically measured at ten meters above the ocean's surface), denoted $\left|U_{w}\right|$, increases. 


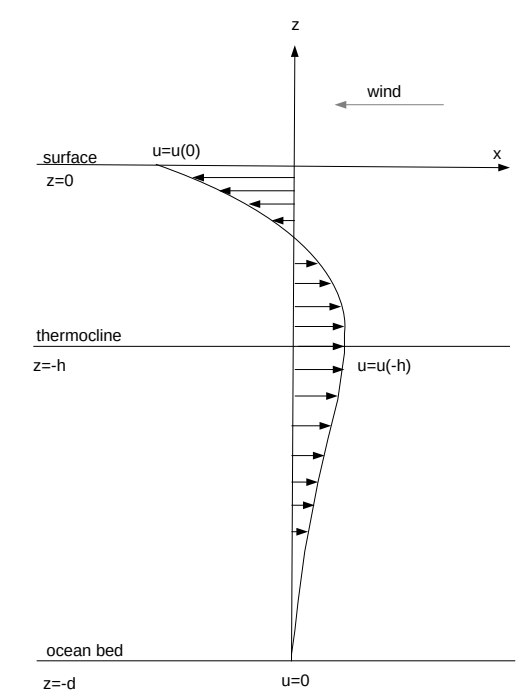

Fig. 1. Typical vertical profile of the equatorial current field in the Pacific region. The horizontal axis points from West to East, along the Equator

Proof. From (2.12) and (2.13) we see that

$$
u_{0}=-\frac{1}{\rho \sigma}\left(\int_{-h}^{0} \frac{1+\frac{s}{h}}{f(s / d)} d s+\frac{1}{1+r} \int_{-d}^{-h} \frac{1+\frac{s}{h}}{f(s / d)} d s\right)<0 .
$$

Let us set $\xi:=\left|u_{0}\right|$, that is, we look at the strength of the flow at the free surface. A calculation shows that

$$
\frac{d \xi}{d h}=\frac{1}{\rho \sigma}\left(-\frac{1}{h^{2}} \int_{-h}^{0} \frac{s d s}{f(s / d)}-\frac{1}{h^{2}(1+r)} \int_{-d}^{-h} \frac{s d s}{f(s / d)}\right)>0
$$

relation that shows that $\xi$ is an invertible function of $h$, from which we can conclude that $h$ is a function of $\xi$ and

$$
\frac{d h}{d \xi}=\left(\frac{d \xi}{d h}\right)^{-1}>0
$$

Denote now $\beta:=-U_{w}$, that is, $\beta$ stands for the strength of the wind at ten meters above the ocean surface. From the relation (2.8) we have that

$$
\beta=\frac{1}{k} \xi \ln \left(\frac{10 g}{a \xi^{2}}+1\right)
$$

We have first that

$$
\frac{d \beta}{d \xi}=\frac{1}{k}\left[\ln \left(\frac{10 g}{a \xi^{2}}+1\right)-\frac{20 g}{10 g+a \xi^{2}}\right]
$$


To determine the sign of the above expression we see first that $\frac{20 g}{10 g+a \xi^{2}}<2$ and therefore $e^{\frac{20 g}{10 g+a \xi^{2}}}<$ $e^{2}$, for all $\xi>0$. On the other hand notice that

$$
e^{2}<\frac{10 g}{a \xi^{2}}+1 \quad \text { for } \quad \xi^{2}<\frac{10 g}{a\left(e^{2}-1\right)} \cong 829.966(m / s) .
$$

Since the latter inequality is equivalent to $\xi<28.80(\mathrm{~m} / \mathrm{s})$, we now see that for $\xi<28.80$ the bracket on the right hand side of (2.17) is strictly positive, thus the function $\beta$ is invertible and the derivative of the inverse is $\frac{d \xi}{d \beta}=\left(\frac{d \beta}{d \xi}\right)^{-1}>0$ and the interval of definition for $\xi$ as a function of $\beta$ is $\left(0, \beta_{\max }\right)$ where $\beta_{\max }:=\beta(28.80)$.

From the previous considerations we see that $h$ can be thought as a function of $\beta$ and

$$
\frac{d h}{d \beta}=\frac{d h}{d \xi} \frac{d \xi}{d \beta}>0
$$

The latter inequality means that if the strength of the wind at ten meters above the ocean surface increases then $h$ increases, therefore, the level of the thermocline, $-h$, decreases.

Remark 2.4. The value of $\beta_{\max }$ computed from formula (2.16) is approximately $518.54 \mathrm{Km} / \mathrm{h}$. According to the World Meteorological Organization, "the record of wind gusts not related to tornados registered to date is $408 \mathrm{~km} / \mathrm{h}$ during Tropical Cyclone Olivia on 10 April 1996 at Barrow Island, Australia". Thus, our considerations accommodate even stronger storms.

We conclude by a monotonicity result concerning the current at the thermocline $u(-h)$ as a function of the strength of the wind above the surface.

Proposition 2.2. The strength of the current at the thermocline decreases as the strength of the wind above the ocean's surface increases. On the other hand, the difference $u(-h)-u(0)$, measuring the strength of the flow reversal, increases as $\left|U_{w}\right|$ increases.

Proof. The first assertion emerges using (2.18), the relation

$$
\frac{d(u(-h))}{d \beta}=\frac{d(u(-h))}{d h} \frac{d h}{d \beta}
$$

and since

$$
\frac{d(u(-h))}{d h}=\frac{1}{(1+r) \rho \sigma} \frac{1}{h^{2}} \int_{-d}^{-h} \frac{s d s}{f(s / d)} d s<0 .
$$

The second assertion follows from

$$
u(-h)-u(0)=\frac{1}{\rho \sigma} \int_{-h}^{0} \frac{1+\frac{s}{h}}{f(s / d)} d s
$$

and since

$$
\frac{d}{d h}\left(\int_{-h}^{0} \frac{1+\frac{s}{h}}{f(s / d)} d s\right)=-\frac{1}{h^{2}} \int_{-h}^{0} \frac{s d s}{f(s / d)}>0
$$

Acknowledgements: We wish to thank the referee for the useful comments and suggestions. 


\section{References}

[1] M. A. Bourassa, D. G. Vincent and W. L. Wood, A Flux Parameterization Including the Effects of Capillary Waves and Sea State, J. Atmospheric Sciences, Vol. 56, (1999), 1123-1139.

[2] A. Constantin, On the modelling of equatorial waves, Geophys. Res. Lett. 39 (2012), L05602.

[3] A. Constantin, An exact solution for equatorially trapped waves, J. Geophys. Res. 117, (2012), C05029.

[4] A. Constantin and P. Germain, Instability of some equatorially trapped waves, J. Geophys. Res.: Oceans 118 (2013), 2802-2810.

[5] A. Constantin, Some nonlinear, equatorially trapped, nonhydrostatic internal geophysical waves, J. Phys. Oceanogr. 44, No.2, (2014), 781-789.

[6] A. Constantin and R. S. Johnson, The dynamics of waves interacting with the Equatorial Undercurrent, Geophysical and Astrophysical Fluid Dynamics, 109, No.4, (2015), 311-358.

[7] A. Constantin and R. Ivanov, A Hamiltonian approach to wave-current interactions in two-layer fluids, Physics of Fluids 27 (2015), 086603.

[8] M. F. Cronin and W. S. Kessler, Near-surface shear flow in the tropical Pacific cold tongue front, J. Phys. Oceanogr. 39 (2009), 1200-1215.

[9] B. Cushman-Roisin and J.-M. Beckers, Introduction to Geophyiscal Fluid Dynamics:Physical and Numerical Aspects, Academic Press, Waltham, MA, 2011.

[10] A. M. Davies, Modelling storm surge current structure. In Offshore and Coastal Modelling, edited by P.P.G. Dyke, A.O. Moscardini and E.H. Robson, (Wiley: New York, 2013), pp. 55-81.

[11] A. V. Fedorov and J. N. Brown, Equatorial waves. In Encyclopedia of ocean sciences, edited by J. Steele, (Academic Press: New York, 2009), 3679-3695.

[12] I. Gallagher and L. Saint-Raymond, On the influence of the Earth's rotation on geophysical flows, "Handbook of Mathematical Fluid Mechanics", Vol. 4 (Eds. S. Friedlander and D. Serre), pp. 201-329, North-Holland, Amsterdam, 2007.

[13] H. García-Nava, F. J. Ocampo-Torres, P. Osuna, and M. A. Donelan, Wind stress in the presence of swell under moderate to strong conditions, J. Geophysical Research, Vol. 114 C12008, doi:10.1029/2009JC005389, 2009.

[14] T. Gerkema, J. T. F. Zimmerman, L. R. M. Maas, and H. van Haren, Geophysical and astrophysical fluid dynamics beyond the traditional approximation, Rev. Geophys. 46 (2004), 1-33.

[15] D. Henry, An exact solution for equatorial geophysical water waves with an underlying current, Eur. J. Mech. B/Fluids 38 (2013), 18-21.

[16] D. Henry and H.-C. Hsu, Instability of equatorial water waves in the f-plane, Discrete Contin. Dyn. Syst. 35 (2015), No.3, 909-916.

[17] D. Ionescu-Kruse, An exact solution for geophysical edge waves in the f-plane approximation, Nonlinear Anal. Real World Appl. 24 (2015), 190-195.

[18] W. S. Kessler and M. J. McPhaden, Oceanic equatorial waves and the 1991-1993, El Niño, J. Climate 8 (1995), 1757-1774.

[19] P. H. LeBlond and L. A. Mysak, Waves in the ocean, (Elsevier, Amsterdam, 1978).

[20] J. P. McCreary, Modeling equatorial ocean circulation, Ann. Rev. Fluid Mech. 17 (1985), 359-409.

[21] J. A. Proehl, M. J. McPhaden and L. M. Rothstein, A numerical approach to equatorial oceanic wavemean flow interactions. In Advanced physical oceanographic modelling, edited by J.J. O'Brien, (D. Reidel Publishing Company: Dordrecht, 1986), pp. 111-126.

[22] H. Stommel, Wind drift near the equator, Deep-Sea Res. 6 (1960), 298-302.

[23] J. O. Wenegrat, M. J. McPhaden and R. C. Lien, Wind stress and near-surface shear in the equatorial Atlantic Ocean, Geophys. Res. Lett. 41 (2014), 1226-1231. 